\title{
Excitation of higher-order modes in optofluidic hollow-core photonic crystal fiber
}

Philipp Köhler, Andrei Ruskuc, Marius A. Weber, Michael H. Frosz, Ana Andres-Arroyo, et al.

Philipp Köhler, Andrei Ruskuc, Marius A. Weber, Michael H. Frosz, Ana Andres-Arroyo, Philip St. J. Russell, Tijmen G. Euser, "Excitation of higherorder modes in optofluidic hollow-core photonic crystal fiber," Proc. SPIE 10744, Laser Beam Shaping XVIII, 107440N (14 September 2018); doi: 10.1117/12.2320594

SPIE Event: SPIE Optical Engineering + Applications, 2018, San Diego, California, United States 


\title{
Excitation of higher-order modes in optofluidic hollow-core photonic crystal fiber
}

\author{
Philipp Koehler ${ }^{\mathrm{a},{ }^{,},}$Andrei Ruskuc ${ }^{\mathrm{a}, \mathrm{b}}$, Marius A. Weber ${ }^{\mathrm{a}}$, Michael H. Frosz ${ }^{\mathrm{c}}$, Ana Andres-Arroyo ${ }^{\mathrm{a}}$, \\ Philip St.J. Russell ${ }^{\mathrm{c}}$, and Tijmen G. Euser ${ }^{\mathrm{a}}$ \\ ${ }^{a}$ NanoPhotonics Centre, Cavendish Laboratory, Department of Physics, University of Cambridge, JJ \\ Thomson Ave, Cambridge CB3 0HE, UK, ${ }^{\mathrm{b}} \mathrm{T}$. J. Watson Laboratory of Applied Physics, California \\ Institute of Technology, 1200 E California Blvd. MC 128-95, Pasadena, CA 91125, USA, ${ }^{\mathrm{C}} \mathrm{Max}$ \\ Planck Institute for the Science of Light, Staudtstr. 2, 91058 Erlangen, Germany
}

\begin{abstract}
Higher-order modes are controllably excited in water-filled kagomè-, bandgap-style, and simplified hollow-core photonic crystal fibers (HC-PCF). A spatial light modulator is used to create amplitude and phase distributions that closely match those of the fiber modes, resulting in typical launch efficiencies of 10-20\% into the liquid-filled core. Modes, excited across the visible wavelength range, closely resemble those observed in air-filled kagomè HC-PCF and match numerical simulations. These results provide a framework for spatially-resolved sensing in HC-PCF microreactors and fiber-based optical manipulation.
\end{abstract}

Keywords: Photonic crystal fibers, Microstructured fibers, Spatial light modulators

\section{INTRODUCTION}

The controlled excitation of higher-order fiber modes has become an essential part in photonics research with a range of interdisciplinary applications. For example, spatial light modulator (SLM)-based wavefront shaping techniques [1] have enabled the controlled excitation of coherent mode superpositions in multimode fibers [2], with novel applications in lensless endoscopic imaging [2]-[4] and fiber-based optical trapping [5]. In fiber communication systems, mode-division multiplexing has been used to improve data transfer rates [6]-[9].

All this previous work aims to control the light field at the end-face of glass-core fibers. In hollow waveguides, on the other hand, well-defined modal intensity distributions can be used to study light-matter interactions within the core. In particular, hollow-core photonic crystal fiber (HC-PCF) has enabled the stable and low-loss transmission of modes along microchannels [10]. The main classes of HC-PCF include bandgap-type HC-PCFs, in which a narrow transmission window is supported by the formation of photonic bandgaps in the microstructured cladding, and kagomé- and simplified HC-PCFs [11], whose broadband guidance mechanism relies on anti-resonant reflection. It has previously been shown that spatial light modulators (SLM) can be used to dynamically change between different modes in air-filled hollow-core photonic crystal fibers (HC-PCFs) [12], with applications in optical trapping [13], Raman amplification [14], telecoms [15], and quantum optics [16].

Here we extend this work to liquid-filled HC-PCFs, where guidance properties are preserved by infiltrating both the core and cladding channels [17]-[18]. Control over modal fields within these optofluidic waveguides would enable new fiberbased sensing and optical manipulation approaches.

*pk428@cam.ac.uk; www.np.phy.cam.ac.uk/research-themes/optofluidics

Laser Beam Shaping XVIII, edited by Angela Dudley, Alexander V. Laskin, Proc. of SPIE Vol. 10744

$107440 \mathrm{~N} \cdot$ C 2018 SPIE · CCC code: 0277-786X/18/\$18 · doi: 10.1117/12.2320594 


\section{EXPERIMENTAL SETUP}

We employ a method based on a spatial light modulation scheme recently presented by Flamm et al. [18] to controllably excite higher order modes into the liquid-filled hollow-core photonic crystal fibers (HC-PCFs). This is achieved by creating an intensity and phase distribution [20] that matches the HC-PCF mode and projecting it onto the fiber's end face. In Section A of Figure 2, light from a supercontinuum laser (NKT SuperK Compact, 450-2400 nm) is passed through a variable bandpass filter (NKT SuperK Varia, 400-840 nm), expanded and linearly polarized. A $30 \mathrm{~cm}$ long HC-PCF is mounted between two custom-made pressure cells (PCs), that are fitted with sapphire windows allowing for unobstructed optical access (Section C). A phase-only SLM (Meadowlark P512-480-850-DVI-C512x512) with broadband mirror coating shapes the beam and projects it in a 4-f configuration onto the fiber (Section B). Cam 2 measures the back-reflected light to help with the alignment process. With a microscope objective the transmitted mode is imaged onto Cam 3 (Section C). Cam1, in Section D, is used to verify the SLM generated intensity profiles, see examples in Figure 3.

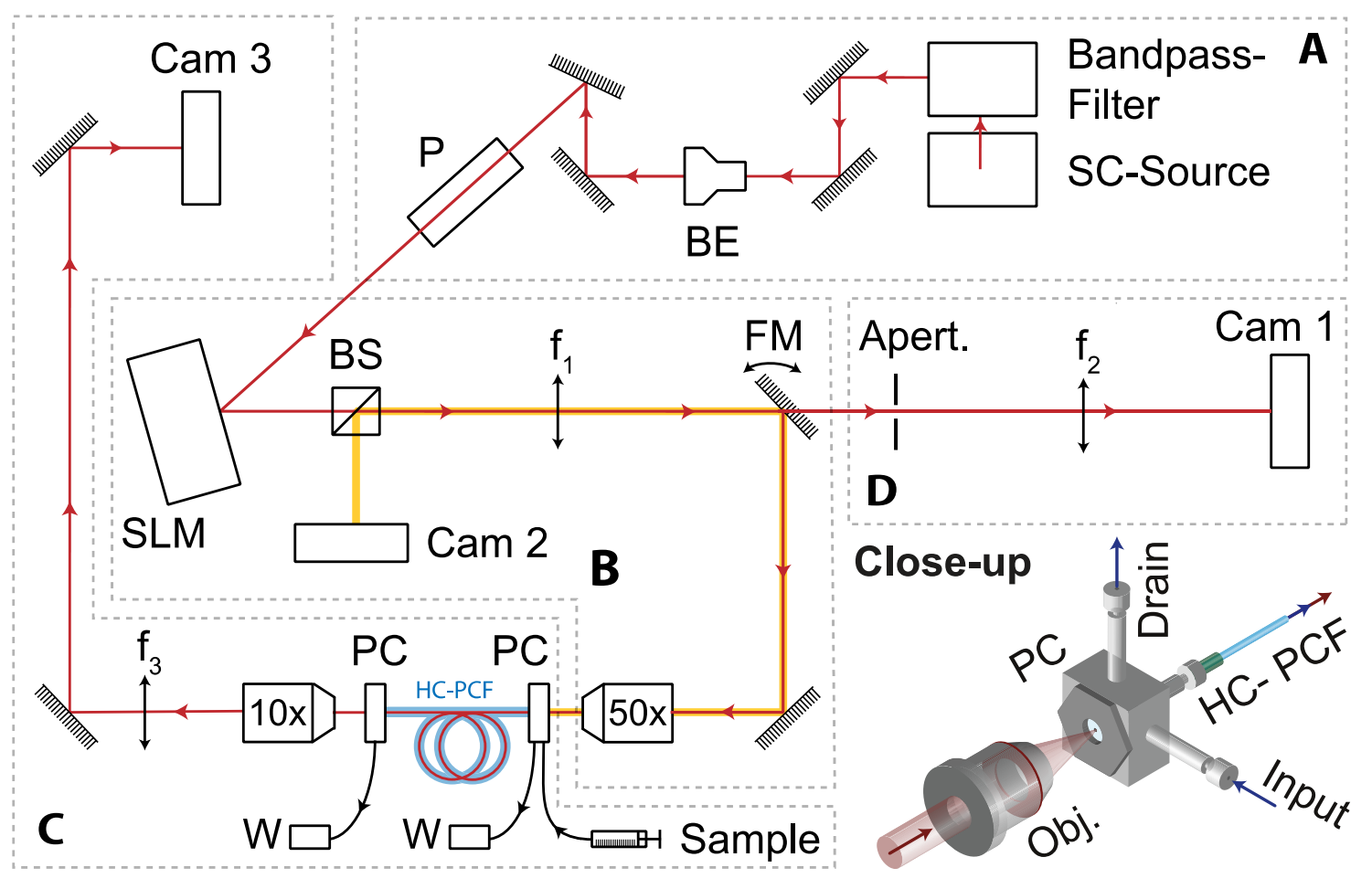

Figure 1. Setup schematic. Section A: filtering, expansion, and polarization of the input beam. Section B: modulation by phase-only SLM and projection onto the input-face of an HC-PCF. Section C: imaging of the end-face of the liquidfilled HC-PCF, enclosed by two pressure cells (PC). Section D: verification of the intensity distribution projected onto the HC-PCF. BE, beam expander; BS, beam splitter; Cam, camera; FM, flip mirror; Apert., aperture; P, polarizer; W, waste. Figure reproduced from [21]. 


\section{MODE EXCITATION}

Efficient mode excitation was achieved with Laguerre-Gaussian beams $\left(\mathrm{LG}_{\mathrm{p}}^{(\ell)}\right)$. The electric field distribution in the focus of an LG beam is given by [22]:

$$
E_{\mathrm{p}}^{(\ell)}(r, \phi) \sim e^{-r^{2} / w^{2}}\left(\frac{r}{w}\right)^{|\ell|} L_{\mathrm{p}}^{|\ell|}\left(\frac{2 r^{2}}{w^{2}}\right) e^{i \phi \ell},
$$

where $\ell$ and $\mathrm{p}$ denote the azimuthal and radial order of the modes respectively, $L_{\mathrm{p}}^{(|\ell|)}$ are the generalized Laguerre polynomials, $r$ and $\phi$ are polar coordinates in the focal plane and $w$ is the beam waist. To excite a specific mode, pairs of LG beams with an appropriate relative phase were chosen. For example, the predicted LP ${ }_{31}$ mode (Fig. 2a) is well approximated by a superposition of $\mathrm{LG}_{0}^{(3)}$ and $\mathrm{LG}_{0}^{(-3)}$ beams (Fig. 2b). Mode-excitation experiments were performed in three different water-filled HC-PCFs including the bandgap HC-PCF, the kagomé HC-PCF, and the simplified HC-PCF. Figure 3 shows the measured intensity distribution of an $\mathrm{LP}_{11}$ mode excitation in each one of these fibers. Additional excited modes and a more detailed analysis can be found in [21].

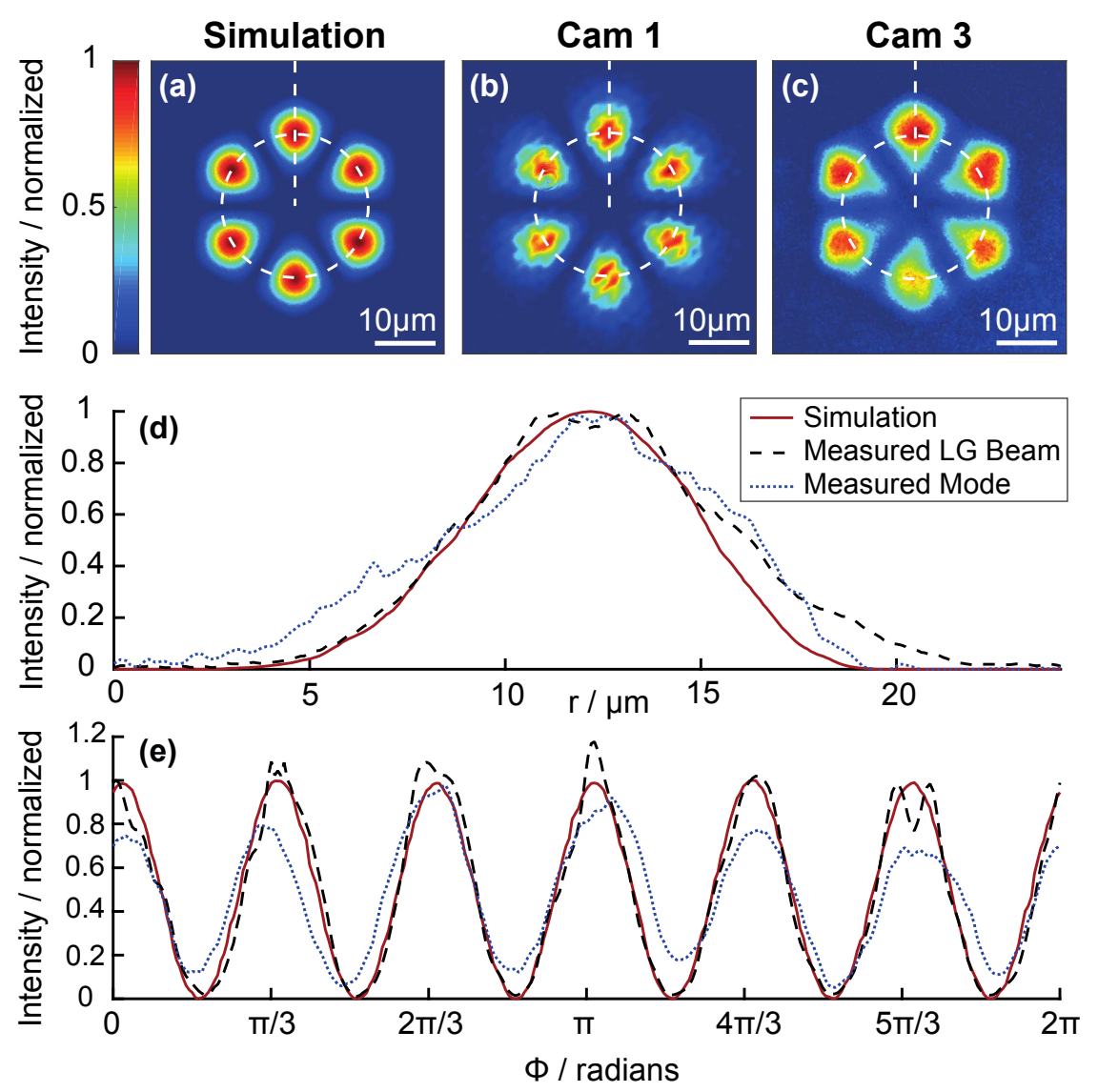

Figure 2. Mode excitation example: (a) Simulated intensity profile of a $\mathrm{LP}_{31}$ core mode in the kagomé PCF. (b) Measured

intensity of an $\mathrm{LG}^{(3)}+\mathrm{LG}^{(-3)}$ beam profile. (c) Measured intensity profile of the excited $\mathrm{LP}_{31}$ fiber mode. Radial- (d) and azimuthal (e) sections along the dashed curves in $(\mathrm{a}-\mathrm{c})$. Figure reproduced from [21]. 


\section{Photonic bandgap HC-PCF}
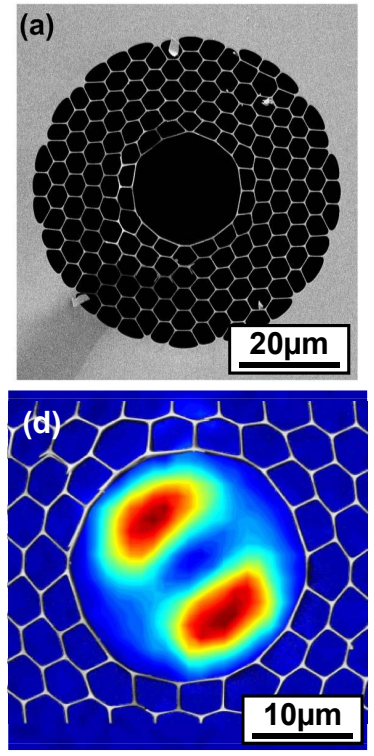

Kagome HC-PCF
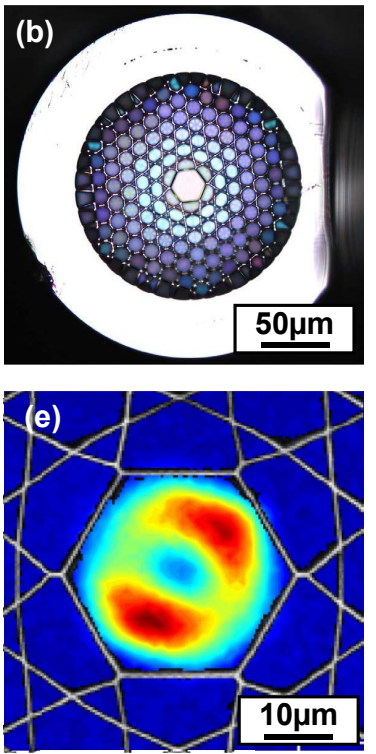

\section{Simplified HC-PCF}
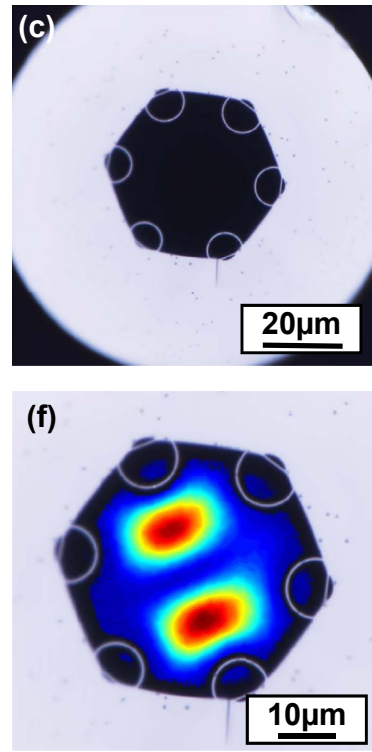

Figure 3. Mode excitation in fibre: Measured intensity profile of a $\mathrm{LP}_{11}$ core mode (d)-(f) in the photonic bandgap HCPCF, kagomé HC-PCF and simplified HC-PCF (a)-(c).

\section{CONCLUSION AND OUTLOOK}

We demonstrate a spatial light modulation setup that can be used to efficiently excite higher-order modes in liquid-filled HC-PCFs. The setup was tested on three different types of water-filled HC-PCFs (bandgap, kagomé, and simplified). While the observed modes were relatively pure and launch efficiencies high (10-20\%), further improvements could be made by correcting for aberrations in the optical system and using a more robust hologram optimization routine.

The results provide a framework for new spatially-resolved sensing and optical manipulation experiments in liquid-filled hollow-core PCF. Measurements using different spatial modes would enable the probing of chemicals at varying distances from the core wall and thus provide a direct measurement of surface effects and microscale diffusive transport, both of which are rate-limiting factors in HC-PCF microreactors [23] and flow-chemistry in general. In optical manipulation studies, superpositions of higher-order modes can be used to create reconfigurable 3-D intensity patterns within the hollow core [13] that could be used to trap, transport, and separate micro- and nanoparticles along the fluid channel.

\section{REFERENCES}

[1] I. M. Vellekoop and A. P. Mosk, "Focusing coherent light through opaque strongly scattering media," Opt. Lett. 32, 2309-2311 (2007).

[2] T. Čižmár and K. Dholakia, “Exploiting multimode waveguides for pure fibre-based imaging,” Nat. Commun. 3, 1027 (2012).

[3] Y. Choi, C. Yoon, M. Kim, T. D. Yang, C. Fang-Yen, R. R. Dasari, K. J. Lee, and W. Choi, "Scanner-Free and Wide-Field Endoscopic Imaging by Using a Single Multimode Optical Fiber,” Phys. Rev. Lett. 109, 203901 (2012).

[4] L. V. Amitonova, A. Descloux, J. Petschulat, M. H. Frosz, G. Ahmed, F. Babic, X. Jiang, A. P. Mosk, P. St.J. Russell, and P. W. H. Pinkse, "High-resolution wavefront shaping with a photonic crystal fiber for multimode fiber imaging," Opt. Lett. 41, 497-500 (2016). 
[5] I. T. Leite, S. Turtaev, X. Jiang, M. Šiler, A. Cuschieri, P. St.J. Russell, and T. Čižmár, “Three-dimensional holographic optical manipulation through a high-numerical-aperture soft-glass multimode fibre," Nat. Photonics 12, 33-39 (2018).

[6] N. Bozinovic, S. Golowich, P. Kristensen, and S. Ramachandran, "Control of orbital angular momentum of light with optical fibers," Opt. Lett. 37, 2451-2453 (2012).

[7] D. J. Richardson, J. M. Fini, and L. E. Nelson, "Space-division multiplexing in optical fibres," Nat. Photonics 7, 354-362 (2013).

[8] R. G. H. van Uden, R. A. Correa, E. A. Lopez, F. M. Huijskens, C. Xia, G. Li, A. Schülzgen, H. de Waardt, A. M. J. Koonen, and C. M. Okonkwo, "Ultra-high-density spatial division multiplexing with a few-mode multicore fibre," Nat. Photonics 8, 865-870 (2014).

[9] H. Huang, G. Milione, M. P. Lavery, G. Xie, Y. Ren, Y. Cao, N. Ahmed, T. A. Nguyen, D. A. Nolan, M.-J. Li, M. Tur, R. R. Alfano, and A. E. Willner, "Mode division multiplexing using an orbital angular momentum mode sorter and MIMO-DSP over a graded-index few-mode optical fibre," Scient. Rep. 5, 14931 (2015).

[10] R. F. Cregan, B. J. Mangan, J. C. Knight, T. A. Birks, P. St.J. Russell, P. J. Roberts, and D. C. Allan, "SingleMode Photonic Band Gap Guidance of Light in Air,” Science 285, 1537-1539 (1999).

[11]P. Uebel, M. C. Günendi, M. H. Frosz, G. Ahmed, N. N. Edavalath, J.-M. Ménard, and P. St.J. Russell, "Broadband robustly single-mode hollow-core PCF by resonant filtering of higher-order modes," Opt. Lett. 41, 1961-1964 (2016).

[12] T. G. Euser, G. Whyte, M. Scharrer, J. S. Y. Chen, A. Abdolvand, J. Nold, C. F. Kaminski, and P. St.J. Russell, "Dynamic control of higher-order modes in hollow-core photonic crystal fibers," Opt. Express 16, 1797217981 (2008).

[13] O. A. Schmidt, T. G. Euser, and P. St.J. Russell, "Mode-based microparticle conveyor belt in air-filled hollowcore photonic crystal fiber," Opt. Express 21, 29383-29391 (2013).

[14] B. M. Trabold, A. Abdolvand, T. G. Euser, A. M. Walser, and P. St.J. Russell, Amplification of higher-order modes by stimulated Raman scattering in H2-filled hollow-core photonic crystal fiber," Opt. Lett. 38, 600-602 (2013).

[15]F. Poletti, N. V. Wheeler, M. N. Petrovich, N. Baddela, E. N. Fokoua, J. R. Hayes, D. R. Gray, R. Li Z., Slavik, and D. J. Richardson, Towards high-capacity fibre-optic communications at the speed of light in vacuum," Nat. Photonics 7, 279-284 (2013).

[16] G. Epple, N. Y. Joly, T. G. Euser, P. St.J. Russell, and R. Löw, Effect of stray fields on Rydberg states in hollow-core PCF probed by higher-order modes," Opt. Lett. 42, $3271\{3274$ (2017).

[17] T. A. Birks, D. M. Bird, T. D. Hedley, J. M. Pottage, and P. St.J. Russell, Scaling laws and vector effects in bandgap-guiding fibres," Opt. Express 12, 69-74 (2004).

[18] G. Antonopoulos, F. Benabid, T. A. Birks, D. M. Bird, J. C. Knight, and P. St.J. Russell, Experimental demonstration of the frequency shift of bandgaps in photonic crystal fibers due to refractive index scaling," Opt. Express 14, 3000-3006 (2006).

[19] D. Flamm, C. Schulze, D. Naidoo, S. Schroter, A. Forbes, and M. Duparre, All-Digital Holographic Tool for Mode Excitation and Analysis in Optical Fibers," J. Lightwave Technol. 31, 1023-1032 (2013).

[20] V. Arrizon, U. Ruiz, R. Carrada, and L. A. Gonzalez, Pixelated phase computer holograms for the accurate encoding of scalar complex fields," J. Opt. Soc. Am. A 24, 3500-3507 (2007).

[21] A. Ruskuc, P. Koehler, M. A. Weber, A. Andres-Arroyo, M. H. Frosz, P. St.J. Russell, T. G. Euser, "Excitation of higher-order modes in optofluidic photonic crystal fiber", Arxiv, https://arxiv.org/abs/1807.08806 (2018).

[22] L. Allen, M. Padgett, and M. Babiker, IV. The Orbital Angular Momentum of Light, vol. 39 of Progress in Optics (Elsevier, 1999).

[23] A. M. Cubillas, S. Unterkofler, T. G. Euser, B. J. M. Etzold, A. C. Jones, P. J. Sadler, P. Wasserscheid, and P. St.J. Russell, "Photonic crystal fibres for chemical sensing and photochemistry," Chem. Soc. Rev. 42, 8629 (2013). 